\title{
Envelhecimento do Processamento Temporal Auditivo
}

\author{
Vera Tôrres das Neves ${ }^{2}$ \\ Universidade Federal do Rio Grande e Universidade de Brasília \\ Maria Angela Guimarães Feitosa \\ Universidade de Brasília
}

\begin{abstract}
RESUMO - O presente artigo faz uma revisão breve da literatura sobre envelhecimento auditivo, abordando os estudos sobre o envelhecimento do processamento temporal auditivo, especificamente, estudos sobre detecção de interrupções em sons, por sujeitos adultos de mais idade. São apresentadas definições e descrições da presbiacusia, suas consequiências, e sua prevalência. São descritos os procedimentos experimentais para estudo de processamento temporal envolvendo a detecção de interrupções em ruídos com faixas amplas de freqüência, a discriminação de sons com reversão temporal, a detecção de mudanças na amplitude de sons, a detecção de interrupções em sons com faixas estreitas de freqüências, a detecção de diferenças de duração entre dois estímulos, bem como a discriminação da ordem temporal de diferentes canais de freqüência componentes de tons complexos. São revisados, adicionalmente, estudos que descrevem as características psicofísicas do processamento auditivo temporal em idosos. Finalmente, são apresentadas sugestões sobre direções futuras para pesquisa.
\end{abstract}

Palavras-chave: envelhecimento auditivo; processamento temporal auditivo; psicofísica da audição; detecção de interrupções no som.

\section{Aging of Temporal Auditory Processing}

\begin{abstract}
The present article contains a brief review of the literature concerning auditory aging, describing studies on the effects of aging on temporal auditory processing, and more specifically, studies about the detection of gaps in sounds, by aging subjects. Definitions, descriptions, consequences and prevalence of presbycusis are discussed. Experimental procedures for research on temporal processing involving the detection of gaps in broadband noises, discrimination of sounds with temporal reversal, detection of sound amplitude changes, detection of gaps on narrowband sounds, detection of differences of duration between two stimuli, as well as the discrimination of the temporal order of different frequency channels composing complex tones are described. Additionally, studies describing the psychophysical characteristics of temporal auditory processing in elderly subjects are reviewed. Finally, suggestions about future directions of research are presented.
\end{abstract}

Key words: auditory aging; auditory temporal processing; auditory psychophysics; gap detection.

No decorrer do século XX, ocorreu uma clara mudança no perfil demográfico da maioria das populações, sendo observado um aumento da longevidade na maior parte do planeta. O envelhecimento demográfico (definido como um aumento da porcentagem da população contando 65 anos de idade ou mais) é uma tendência estabelecida em todas as regiões do planeta, com exceção da África (United Nations, 1994). Até o momento, este processo tem sido mais evidente nos países desenvolvidos; contudo, as projeções sobre o envelhecimento populacional para a Ásia e a América Latina para 2025 apontam níveis de mudança semelhantes aos que ocorreram na Europa na década de 1970. O envelhecimento de uma população usualmente é consequiência da passagem de um país para o estágio final de uma transição demográfica em que ocorre uma queda duradoura nas taxas de fertilidade (Chesnais, 1992). À medida que diminui o número de crianças nascidas nos grupos populacionais mais jovens, aumenta a importância relativa da população mais

1 Durante a realização do presente trabalho a primeira autora contou com bolsa PICD da CAPES e a segunda autora com bolsa de produtividade do $\mathrm{CNPq}$.

2 Endereço: Universidade de Brasília, Instituto de Psicologia, Depto de Processos Psicológicos Básicos, 70910-900 Brasília DF. E-mails: vtn@terra.com.brou afeitosa@unb.br idosa. Este efeito é amplificado por uma significativa redução na mortalidade entre os adultos, que contribui para um aumento da expectativa de vida (Lloyd-Sherlock, 2000).

Nos países desenvolvidos a visão tradicional do idoso, como alguém pobre, com pouca educação, com saúde frágil e declinante, tem sido superada em função do aumento dos níveis de educação e de prosperidade decorrentes do progresso científico e tecnológico atual. Contudo, isto não impede que o envelhecimento, mesmo nestes países, seja o principal correlato de doenças e incapacitação. Portanto, ainda no século 21, o idoso típico apresentará diversos problemas fisiológicos, como diabetes, hipertensão arterial, insuficiência cardíaca e renal, demência, redução da mobilidade, quedas e fraturas decorrentes de osteoporose (Hazzard, Woolard \& Regenstreif, 2001). Recentemente, por exemplo, Wyn e Solis (2001) verificaram que $31 \%$ das mulheres norte-americanas com mais de 65 anos de idade apresenta uma incapacitação ou problema de saúde que limita sua participação em atividades diárias. Além dos, já mencionados, problemas de hipertensão, cardiopatias, diabetes e osteoporose, são significativas, também, as ocorrências de câncer, artrite, depressão e ansiedade (17\% das mulheres idosas recebem tal diagnóstico em 5 anos e um terço apresentam relatos de sintomas depressivos). 
Em função de tais características do envelhecimento, atualmente, diversas investigações têm sido conduzidas com o propósito de se identificar os determinantes da ocorrência de doenças crônicas e incapacitação em idosos. Nestes estudos, procura-se verificar, em termos gerais, que aspectos da saúde ou da vida quotidiana de idosos tornam tais pessoas mais propensas a se tornarem dependentes de familiares ou instituições assistenciais. Em alguns estudos, verifica-se que características pessoais, como nível sócio-econômico, região geográfica de residência e ocorrência de eventos vitais estressantes têm efeito sobre o estado de saúde dos idosos (Grundy \& Holt, 2000). Por outro lado, estudos como o de Leinonen, Heikkinen e Jylhä (2001) constatam que pessoas idosas (entre 75 e 80 anos) tendem a ter uma avaliação subjetiva de seu estado geral de saúde bastante estável com o avançar da idade (tendem a considerar sua saúde consistentemente boa, razoável ou precária, em termos gerais, com o decorrer dos anos), apesar de serem capazes de indicar com precisão a ocorrência de mudanças objetivas em sua saúde neste período, como o surgimento de novas doenças, ou o agravamento de problemas já existentes. Entretanto, as autoavaliações do estado geral de saúde tendiam a piorar quando estas pessoas sofriam redução de sua capacidade física, sua sensibilidade auditiva (limiar absoluto de audição), sua capacidade cognitiva (escores no subteste digit-symbol da Wechsler Adult Intelligence Scale) ou sua atividade física (tornando-se mais sedentárias), ou quando surgiam novas doenças crônicas. Adicionalmente, apontavam mudanças negativas de saúde quando, além das ocorrências listadas acima, aumentavam as suas dificuldades para executar atividades da vida diária (como se alimentar, se vestir, cuidar da própria higiene ou deambular dentro ou fora de casa).

Além dos problemas de saúde física, idosos têm também um aumento da prevalência de sintomas depressivos (Schoevers \& cols, 2000; Van den Berg \& cols. 2000; Fonda \& Herzog, 2001; Mills, 2001) e, consequentemente, do risco de cometer suicídio (Pearson \& Brown, 2000), além de estarem mais expostos a abuso e negligência por parte de seus familiares e prestadores de cuidados (Giurani \& Hasan, 2000).

\section{Envelhecimento Auditivo}

Como foi visto acima, a capacidade perceptual do idoso e a sua capacidade de desempenhar adequadamente suas atividades cotidianas têm um efeito significativo na auto-avaliação de seu estado de saúde. Dentre os diversos problemas que reduzem a qualidade de vida dos idosos, os distúrbios auditivos estão entre os mais comuns, como apontam diversos estudos epidemiológicos (Willott, 1991; Cruickshanks \& cols., 1998; Morrell, Gordon-Salant, Pearson, Brant \& Fozart, 1996; Kacker, 1997). Nos Estados Unidos, aproximadamente $30 \%$ das pessoas com mais de 65 anos de idade, e $50 \%$ das pessoas com mais de 75 anos de idade afirmam que sofreram alguma perda auditiva (Clark \& cols.1995). Apesar de diferenças metodológicas entre os diversos estudos resultarem em índices de prevalência diferentes, todos permitem algumas conclusões em comum. Em primeiro lugar, é amplamente reconhecido o fato de que a prevalência de problemas auditivos, que podem ser detectados por métodos audiométricos rotineiros, aumenta significativamente com a idade. Cruikshanks e cols. (1998), por exemplo, verificaram que, com o envelhecimento, ocorrem mudanças no nível de audição ${ }^{1}$ médio para as faixas de som de 2,3 e 4 kHz. Na amostra estudada (3.753 pessoas com idades entre 48 e 92 anos de idade), como um todo, a prevalência de perdas auditivas era de $45,9 \%$. Dentre os sujeitos com perdas auditivas, $58,1 \%$ tinham perdas leves (entre 25 e $40 \mathrm{~dB}$ ), $30,6 \%$ tinham perdas moderadas (entre 40 e $60 \mathrm{~dB}$ ) e $11,3 \%$ tinham perdas pronunciadas (acima de $60 \mathrm{~dB})^{2}$. Em segundo lugar, as perdas auditivas começam a ocorrer, em alguns indivíduos, bem antes da idade avançada, já que a elevação em sensibilidade absoluta começa a ocorrer desde a terceira e quarta décadas de vida. E, finalmente, a prevalência de problemas auditivos aumenta com o envelhecimento mais extensamente entre homens do que entre mulheres.

As conseqüências das perdas auditivas em idosos são, evidentemente, adversas. Por exemplo, Visentin, Scarafiotti, Marinello, Molaschi e Fabris (1998) verificaram que, entre 747 pessoas com idade acima de 75 anos, os sintomas de fadiga, perda de memória, problemas de nutrição, indigestão ou problemas de audição ou fala, permitiam identificar aquelas que tinham sofrido perda de capacidade cognitiva, aumento da dependência para executar atividades da vida diária ou aumento do sofrimento psicológico. Resultados semelhantes foram encontrados por Lee, Smith e Kington (1999): as limitações auditivas e visuais se correlacionavam com diminuição da capacidade funcional (para executar atividades da vida diária) em 7.320 pessoas acima de 70 anos de idade, residentes na comunidade, mesmo quando eram controlados os efeitos de fatores demográficos, status socioeconômico e estado geral de saúde. Semelhantemente, Laukkanen, Leskinen, Kauppínen, Sakari-Rantala e Heikkinen (2000) verificaram que a capacidade de $183 \mathrm{mu}-$ lheres, com mais de 80 anos de idade, permanecerem residindo nas suas comunidades, sem sofrer internação em hospitais ou instituições assistenciais era diretamente correlacionada a sua sensibilidade auditiva. Por outro lado, Tsuruoka e colaboradores (2001) verificaram que entre 60 idosos (idades acima de 65 anos), residindo em instituições assistenciais, as perdas auditivas dificultavam suas relações com funcionários e outros residentes, por impedirem sua comunicação, tornando-os retraídos, inseguros e deprimidos. Ou seja, as

1. O nível de audição consiste em uma medida em que a sensibilidade auditiva é comparada à norma populacional, permitindo identificação fácil de perda de audição.

2. O decibel $(\mathrm{dB})$ é uma medida de nível de intensidade do som. Zero decibéis correspondem ao limiar absoluto de detecção de um som, o nível mínimo detectável de um som, na ausência de qualquer outro som externo, ou seja, o limite abaixo do qual um som tipicamente não é perceptível. Trinta decibéis correspondem a um sussurro baixo, e sessenta decibéis é a intensidade da voz a um metro de distância, quando se está falando normalmente. 
perdas auditivas afetavam tanto as comunicações quanto os domínios sociais e emocionais de idosos internados.

Entretanto, como será visto nos estudos a seguir, apesar de já ser amplamente reconhecida a importância do estudo de tais problemas, muito esforço ainda está sendo investido na tarefa de discriminar, adequadamente, quais seriam as características do envelhecimento auditivo "normal", típico da população em geral, e quais indicariam a ocorrência de um processo patológico de perda auditiva. Esta discriminação é dificultada pelo fato de que a exposição crônica a ruído, seja decorrente da exposição a níveis danosos de ruídos específicos (nas atividades industriais ou recreativas) ou a exposição ao ruído ambiental geral (sociocusia), é um dos principais componentes da perda auditiva relacionada ao envelhecimento (presbiacusia), juntamente com a degeneração fisiológica (envelhecimento) e os efeitos de doenças e intoxicações (nosocusia). A audição das pessoas com perdas induzidas por ruídos tende a piorar com a passagem do tempo, mas não é possível identificar-se com certeza quais as contribuições relativas do ruído e do envelhecimento na progressão da perda auditiva. Até o momento, é sabido, apenas, que a progressão da perda auditiva induzida por ruídos tende a desacelerar com a passagem do tempo, enquanto a perda auditiva relacionada à idade tende a acelerar, ocorrendo uma taxa maior de perda para as frequiências ${ }^{3}$ altas e uma perda menor nas frequiências baixas (Gates, Schmid, Kujawa, Nam \& D'Agostino, 2000).

Os termos "presbicusia" e "presbiacusia" têm sido usados para descrever a perda de audição associada ao envelhecimento. Historicamente, tem existido uma tendência a se definir a presbiacusia em termos de elevação de limiares audiométricos. Contudo, durante o envelhecimento podem ser alterados, também, aspectos supraliminares da percepção, como de sonoridade, a localização de sons no espaço, o reconhecimento da fala e a percepção de sinais mascarados por ruídos. Por outro lado, as alterações auditivas associadas ao envelhecimento podem ser causadas por diversas disfunções e patologias, tanto periféricas quanto centrais. Assim, o termo "presbiacusia" refere-se, mais propriamente, a uma variedade de distúrbios de audição associados ao envelhecimento. Uma descrição genérica, razoavelmente abrangente, para este conjunto de distúrbios seria: "uma perda bilateral da audição para sons de alta freqüência, acompanhada, geralmente, por uma perda desproporcional do reconhecimento da fala, sem história prévia de doença sistêmica ou auditiva severa, com início gradual e curso progressivo" (Willott, 1991, p. 2).

\section{Processamento Temporal}

Dentre os vários aspectos do funcionamento auditivo já estudados, um dos menos explorados tem sido o processa-

3. A freqüência corresponde ao número de períodos de uma onda sonora que ocorrem em um segundo. É medida em ciclos por segundo, ou Hertz $(\mathrm{Hz})$. Freqüências altas correspondem a sons mais agudos, e frequiências baixas a sons mais graves. mento temporal auditivo. Moore (1997) o define como sendo a percepção de sons que variam com o tempo, especialmente no que se refere aos limites da capacidade de se detectar mudanças com o tempo. A variável tempo é importante na percepção dos sons como a fala e a música, porque a maior parte da informação que estes sons transportam está contida em suas variações ao longo do tempo. É também importante para alguns aspectos da localização de sons no espaço.

O processamento temporal tem dois aspectos principais: a resolução (ou acuidade) temporal e a integração (ou somação) temporal. Resolução temporal refere-se à capacidade de se detectar mudanças nos estímulos ao longo do tempo, por exemplo, detectar uma interrupção breve entre dois sons, ou detectar alguma forma de modulação em um som. Integração temporal refere-se à capacidade do sistema auditivo de combinar a informação apresentada ao longo de um tempo para aumentar a detecção ou discriminação de estímulos (Moore, 1997).

A resolução temporal depende de dois processos: a análise do padrão temporal que ocorre em cada canal de freqüência (análise temporal intra-canal) e a comparação dos padrões temporais dos vários canais auditivos ativados a cada momento (análise temporal inter-canais). Tais canais se referem às características de filtragem do sistema auditivo periférico. A cóclea comporta-se como um conjunto de filtros (chamados "filtros auditivos"), que separam os componentes de um sinal complexo em "canais", afinados com diferentes freqüências centrais. A análise temporal pode ser considerada como o resultado destes dois processos principais (Moore, 1993). Uma descrição dos principais procedimentos utilizados no exame da resolução temporal foi realizada por Moore (1997) e é resumida a seguir.

\section{Procedimentos para Estudo do Processamento Temporal}

Dentre os processos de análise temporal intra-canal, o mais estudado é o processo de detecção de interrupções em ruídos de faixa ampla de freqüências. Isto porque, o espectro $^{4}$ de magnitudes de longo prazo de ruídos brancos mantém-se constante durante interrupções breves, permitindo que a detecção da interrupção não seja indevidamente facilitada pela ocorrência de mudanças no espectro de magnitude. Geralmente, este processo é estudado através de procedimentos psicofísicos de escolha forçada entre duas alternativas (2AFC), nos quais são apresentados ao sujeito dois jorros sucessivos de som em que o primeiro ou o segundo som (aleatoriamente) é interrompido. A tarefa do sujeito é indicar qual dos dois sons contém a interrupção. Tipicamente, o limiar de detecção de interrupções entre jovens com limiares de audição normal é de 2 a 3 milissegundos (ms) (Plomp,

4. O espectro de uma onda sonora é a distribuição das freqüências que a compõem e suas magnitudes . Pode ser representado com gráficos da amplitude, intensidade ou nível destes componentes em função de suas freqüências. 
1964b; Penner, 1977). Este limiar aumenta com níveis muito baixos de sonoridade, próximos do limiar absoluto, mas é geralmente invariante com níveis moderados ou altos.

Uma segunda forma de se examinar o processamento temporal auditivo envolve a discriminação de sinais com reversão temporal. A magnitude do espectro de longo prazo de um som não é mudada quando este som sofre reversão temporal (é apresentado de trás para diante). Assim, se um som temporalmente revertido pode ser discriminado do som original, isto indica uma sensibilidade à diferença no padrão temporal entre os dois sons. Podem ser usados, por exemplo, um par de cliques diferindo em amplitude ${ }^{5}$. Pede-se que os sujeitos distingam pares que diferem na ordem dos sons, AB ou BA. Em um estudo (Ronken, 1970), os sujeitos conseguiram discriminar pares de tons ${ }^{6}$ separados por intervalos de até 2 ou $3 \mathrm{~ms}$.

Outra maneira de se examinar a resolução temporal consiste em medir o limiar de detecção de mudanças na amplitude de um som em função da rapidez das mudanças. Na sua forma mais simples, um ruído branco sofre uma modulação ${ }^{7}$ senoidal ${ }^{8}$ de sua amplitude e o limiar de detecção da modulação é determinado como uma função da taxa de modulação. Esta função que relaciona o limiar à taxa de modulação é conhecida como função de transferência temporal de modulação (TMTF). Esta medida só se relaciona à resolução temporal para taxas de modulação maiores que $16 \mathrm{~Hz}$, não sendo detectáveis as mudanças acima de $1.000 \mathrm{~Hz}$. Um exemplo deste tipo de estudo foi apresentado por Bacon e Viemeister (1985).

Uma forma mais complexa de estudo de resolução temporal consiste na detecção de interrupções em sons com faixas estreitas de frequiência. Geralmente, a detecção é pior nas faixas de frequiências baixas que nas faixas de freqüências altas. Contudo, aparentemente, a amplitude da faixa de som apresentada parece ser mais decisiva que a frequiência do som em si, dado que uma faixa ampla pode ativar várias partes da cóclea simultaneamente, facilitando a detecção da interrupção (Grose, 1991), O limiar de detecção de interrupções em ruídos com faixas estreitas de frequiência tende a diminuir à medida que aumenta o nível do som, até $30 \mathrm{~dB}$, permanecendo aproximadamente constante a partir deste nível.

Adicionalmente, pode ser examinada a capacidade de se detectar mudanças na duração de estímulos auditivos. Tipicamente, são apresentados dois sons sucessivos com dura-

5. A amplitude de uma onda sonora determina a intensidade com que um som será percebido. Quanto maior a amplitude, mais forte é considerado um som.

6. Um tom puro é uma onda sonora cujas variações instantâneas em função do tempo traçam uma função senoidal. Um tom complexo é um tom composto por uma série de senóides com freqüências diferentes.

7. A modulação consiste em uma mudança em uma determinada dimensão de um som, como sua freqüência ou amplitude. A modulação de frequiência é o processo pelo qual se faz mudar a freqüência de um som em função do tempo

8. Uma onda senoidal é uma forma de onda sonora que varia em função do tempo de acordo com uma função seno. ções diferentes. Pede-se que o sujeito indique qual dos sons tem a maior duração. Creelman (1962) e Abel (1972) verificaram que a menor diferença de duração detectável aumenta com o aumento da duração da linha base. Por exemplo, Abel verificou que quando o tempo de duração do estímulo variava entre 10, 100 e $1000 \mathrm{~ms}$, os aumentos detectados eram de 4, 15 e 60 ms, respectivamente. Divenyi e Danner (1977) verificaram, adicionalmente, que o desempenho era claramente pior quando o nível dos sons era inferior a $25 \mathrm{~dB}$ $\mathrm{SL}^{9}$, ou quando os dois sons diferiam muito em nível ou freqüência. Contudo, para sons com mais de $10 \mathrm{~ms}$ de duração, a freqüência dos sons não afetava a diferença detectável.

A análise temporal baseada em processos inter-canais envolve a comparação de tempo entre diferentes canais de frequiência. Podem ser estudados, por exemplo, a detecção de assincronias entre o início e o fim de componentes de um complexo de sons e o julgamento da ordem temporal de sequiências de sons. O julgamento da ordem temporal de seqüências de sons pode ter limiares muito díspares, dependendo da forma como o sujeito desempenha a tarefa. Quando se pede que o sujeito faça uma discriminação de diversas ordens, a resolução é bastante aguçada. Com treinamento, os sujeitos conseguem distinguir seqüências de sons com menos de 10 ms de duração (Warren, 1974; Divenyi \& Hirsh, 1974). Este é o nível de sensibilidade necessário para se identificar sons da fala. Contudo, quando é necessário identificar a ordem de vários sons em uma seqüência, o desempenho é pior. Por exemplo, Hirsh (1959) verificou que, com pares de sons não relacionados, os sujeitos só faziam identificações corretas diante de sons com, pelo menos, $20 \mathrm{~ms}$ de duração, sendo que quando o número de sons aumentava, sua duração devia ser aumentada também.

\section{Características Psicofísicas do Processamento Auditivo Temporal em Envelhecimento}

Willott (1991) afirma que as perdas auditivas neurossensoriais são freqüentemente associadas a um desempenho precário de tarefas de processamento temporal em sujeitos jovens; consequentemente, a presbiacusia periférica poderia ser um dos elementos determinantes de déficits de processamento temporal em idosos.

Nas tarefas de discriminação temporal, tanto monaurais ${ }^{10}$ quanto binaurais, costuma-se apresentar um par de estímulos aos sujeitos para se determinar qual seu ponto de fusão. É aumentado e diminuído o tempo de separação entre os dois sons, para se verificar qual o intervalo necessário para que sejam percebidos como um só som, ou como dois sons separados. A capacidade de detectar-se intervalos pequenos de silêncio começa a diminuir durante a década dos 50 anos,

9. SL - Sensation Level - é o nível de um som em decibéis em relação ao nível limiar deste som para uma determinada pessoa.

10. Monaural é a condição em que sons são apresentados somente a um ouvido. Binaural é a condição envolvendo audição com os dois ouvidos. 
deteriorando-se progressivamente com o envelhecimento (McCroskey \& Kasten, 1982).

A seguir são revisados alguns estudos que investigam como o envelhecimento afeta o processamento temporal de diversos tipos de som. Estes estudos apresentam como característica comum a adoção de delineamentos envolvendo a comparação do desempenho de ouvintes jovens com ouvintes idosos, com perfis audiológicos semelhantes, incluindo, algumas vezes, também, um grupo de idosos com déficits auditivos, como presbiacusia.

Alguns estudos com idosos examinaram o processamento temporal de tons puros. Assim, Moore, Peters e Glasberg (1992) usaram senóides (com freqüências de 100, 200, 400, $800,1.000$ e $2.000 \mathrm{~Hz}$ ) para comparar o desempenho de jovens e idosos com audição normal e com perdas de audição (idades entre 62 e 86 anos) em tarefas de detecção de interrupções em sons. Os limiares de detecção de interrupções nos níveis mais altos de sonoridade eram semelhantes entre idosos com audição normal ou deficiente. Contudo, a maioria dos idosos, com ou sem perdas auditivas quanto ao limiar absoluto de detecção de sons, apresentava limiares de detecção de interrupções normais, o que indicaria, segundo estes autores, que a redução da resolução temporal não parece ser uma conseqüência inevitável do envelhecimento.

Já outros autores, também utilizando tons puros, chegaram a conclusões diferentes. Assim, Schneider, PichoraFuller, Kowalchuk e Lamb (1994) compararam os limiares de detecção de interrupções em tons com $2 \mathrm{kHz}$ de frequência. Seus sujeitos jovens tinham uma média de idade de 23 anos com um desvio padrão de 1,5 anos, enquanto os idosos tinham em média 69,2 anos com desvio padrão de 4,8 anos. Verificaram que os limiares dos idosos eram duas vezes maiores que os dos jovens, apresentando, também, maior variabilidade. Contudo, tais limiares não se correlacionavam com os limiares audiométricos em qualquer dos dois grupos. Da mesma forma, Strouse, Ashmead, Ohde e Grantham (1998) mediram o processamento temporal monaural e binaural e a percepção de fala em jovens (entre 20 e 30 anos) e idosos (entre 66 e 75 anos de idade) com limiares normais ${ }^{1}$, utilizando tarefas de detecção de interrupções em tons puros com freqüências variando entre 0,25 e 6 kHz, além de tarefas de detecção de intervalo interaural. O desempenho dos idosos foi pior nos três tipos de tarefa, sendo maior o efeito de níveis baixos de sonoridade dos estímulos sobre os idosos. A falta de correlação entre as medidas das três tarefas e o limiar absoluto em idosos sugeriu a estes autores que outros fatores associados ao envelhecimento, além da perda auditiva periférica contribuem para os déficits de processamento temporal dos idosos. Contudo, tal conclusão deve ser avaliada com cautela, dado que os grupos de jovens e idosos não tinham limiares absolutos comparáveis para tons acima de $6 \mathrm{kHz}$.

Provavelmente, as inconsistências entre as conclusões destes estudos decorrem da dificuldade de se investigar processamento temporal com uso de tons puros. Geralmente, o resultado de tais estudos é fortemente afetado pela fase ${ }^{11}$ da senóide em que o som é interrompido e recomeçado
(Shailer \& Moore, 1987). Dependendo da fase em que são feitas estas mudanças, a variação dos limiares de detecção pode não ser monotônica, sendo o aumento ou diminuição do limiar independente da duração do tom, variando em função da fase do tom em que as interrupções ocorrem.

Por outro lado, vários estudos examinaram o desempenho de idosos com utilização de sons mais complexos, como faixas de freqüência de sons, chegando a conclusões mais homogêneas. Assim, Snell (1997), comparou o desempenho de idosos (64 a 77 anos) e jovens (17 a 40 anos) com perfis audiométricos equivalentes em tarefas de detecção de interrupções de ruídos com diversas freqüências superiores de corte $^{12}(1 \mathrm{ou} 6 \mathrm{kHz})$, diferentes níveis de intensidade (70 ou 80 dB SPL ${ }^{13}$ ) e de modulação. Foram verificados efeitos da freqüência do sinal, sua intensidade e idade dos sujeitos sobre o desempenho. Os limiares dos idosos foram mais altos que os dos jovens em todas as condições, indicando que estes tinham uma resolução temporal mais precária. Semelhantemente, He, Horwitz, Dubno e Mills (1999) determinaram funções psicométricas de detecção de interrupções em ruídos de faixa ampla de freqüência ( $5 \mathrm{kHz}$ de amplitude), utilizando um paradigma sim-não, com jovens (média de idade de 31,9 anos e desvio padrão de 8,1 anos) e idosos (média de idade de 70,5 anos e desvio padrão de 5,4 anos) com limiares de audição normal. Apesar de jovens e idosos terem apresentado desempenhos semelhantes na tarefa de detecção de interrupções quando estas ocorriam no meio dos sinais, eqüidistantes de seu início ou fim, foi verificado que os idosos apresentaram limiares de detecção de interrupções mais altos que os jovens diante de estímulos de duração muito curta, ou quando as interrupções estavam muito próximas do início ou fim dos sinais. Este achado é muito relevante quando se compara o desempenho nestas provas com a situação real de detecção de sons de consoantes na linguagem falada. Estes sons se situam freqüentemente no início ou fim das sílabas, o que tornaria sua detecção especialmente difícil para idosos.

Da mesma forma, o desempenho de jovens (média de idade de 21,9 anos, desvio padrão de 1,7 anos) e idosos (média de idade de 72,4 anos e desvio padrão de 5,2 anos) com

11. Fase - é um dos parâmetros que podem ser usados para descrever um movimento vibratório, juntamente com a amplitude, a frequiência e o período. Uma onda tem os ângulos de suas fases variando entre zero e 360 graus. Em zero graus, a onda se encontra no início de seu movimento ascendente, em 90 graus atinge seu ápice, em 180 graus, retorna ao ponto inicial de seu movimento, em 270 graus atinge seu ponto mais baixo, e em 360 graus volta a seu ponto inicial.

12. A freqüência de corte indica um limite acima ou abaixo do qual os componentes de um som complexo são significativamente atenuados. Assim, por exemplo, em um filtro passa-banda, a freqüência de corte superior indica o valor de freqüência acima do qual os sons serão significativamente atenuados, e a freqüência de corte inferior indica o valor da freqüência abaixo do qual o som será significativamente atenuado.

13. SPL - Sound Pressure Level - é o nível de um som em decibéis em relação a um nível de referência internacionalmente definido. Este nível corresponde a uma intensidade de $10^{-12} \mathrm{w} / \mathrm{m}^{2}$, que é equivalente a uma pressão de som de $20 \mu$ Pascal. 
limiares normais foi comparado com o de idosos nos estágios iniciais da presbiacusia, em tarefas de detecção de interrupções em tons complexos (compostos por 22 ou 27 tons puros superpostos), com durações variando entre 0,83 e 500 ms (Schneider \& Hamstra, 1999). Os limiares de detecção dos idosos eram pronunciadamente mais altos para os sinais com durações menores que $250 \mathrm{~ms}$, convergindo com os limiares dos jovens na duração de 500 ms. Para ambas as idades, os limiares de detecção de interrupção eram independentes dos limiares audiométricos.

Conclusões semelhantes foram obtidas por Snell e Frisina (2000), ao compararem o reconhecimento de linguagem com a detecção de interrupções em faixas amplas de freqüências de som. Tais autores examinaram as relações entre as diferenças associadas à idade na detecção de interrupções e reconhecimento de palavras em sujeitos com audição normal ou perda auditiva moderada (jovens com idade entre 17 e 40 anos, média de 26,4 anos; idosos entre 61 e 82 anos, média de 68,3 anos). Diante de ruídos com freqüências superiores de corte de 1 ou $6 \mathrm{kHz}$ e nível geral de $80 \mathrm{~dB}$ SPL, os idosos apresentaram limiares mais altos que os jovens. Contudo tais limiares não se correlacionaram significativamente com os limiares audiométricos para qualquer dos dois grupos. Os autores apontaram a ocorrência de mudanças no processamento auditivo que aconteceriam durante toda a vida adulta, iniciando-se as alterações de sensibilidade temporal décadas antes do surgimento de déficits no reconhecimento de palavras.

Como vimos, grande parte dos estudos revisados encontraram limiares de detecção de interrupções maiores entre idosos do que entre jovens. Estes limiares aumentados não pareciam ter relação com limiares absolutos nem com deficiências auditivas, ocorrendo uma maior variabilidade de resultados entre idosos que entre jovens. Ou seja, o processamento temporal seria uma capacidade auditiva distinta, que poderia, em alguns casos, ser determinado pelos danos comuns aos casos de presbiacusia sensorial ou neuronal, enquanto, em outros casos, poderia ser determinado por outros fenômenos típicos do envelhecimento, independentes dos processos comumente associados à presbiacusia, pelo menos como classicamente definida.

Cabe, entretanto, apontar que os estudos revisados acima carecem de uma abordagem mais exaustiva do processo de mudança do processamento temporal com o envelhecimento, sendo em parte um estudo das características dos idosos e, em parte, um estudo das características do processamento temporal. Nestes estudos, são feitas comparações entre jovens e idosos como grupos isolados, sem que exista uma definição consensual no campo do que constitui a idade que define claramente o início do envelhecimento dos processos de análise temporal de estímulos auditivos. Por isto, observa-se uma grande variação na composição etária dos grupos de idosos examinados entre um estudo e outro. Por outro lado, não se identificou um estudo que procure descrever de maneira contínua como estas capacidades são alteradas com o envelhecimento. Destarte, primeiramente, seria recomendável que fossem realizados estudos em que a idade fosse examinada como uma variável contínua, não dicotomizada entre jovens idosos. Isto permitiria um detalhamento adequado de tais mudanças, identificando, entre outras informações, em que idade tais mudanças se tornam observáveis.

Ademais, a pesquisa básica tem verificado que a diminuição da capacidade de processamento temporal decorrente do envelhecimento nem sempre é correlacionada a danos do aparelho auditivo periférico, podendo correlacionar-se a perdas neuronais centrais próprias da senescência. Ou seja, o processamento temporal pode ser, em grande parte, uma forma de processamento central, executada no encéfalo.

\section{Sugestões de Pesquisas Futuras}

É recomendável que estudos futuros sejam dedicados ao esclarecimento das relações entre a redução da capacidade de processamento temporal, que ocorre com o envelhecimento, e outros aspectos do envelhecimento auditivo, como o aumento do limiar absoluto de audição para sons de altas freqüências. Apesar de existirem evidências de que o processamento temporal seja um fenômeno independente de outros aspectos da audição, como a discriminação de freqüências de som ou o limiar absoluto de audição, as relações entre estes diversos fenômenos ainda não foi suficientemente esclarecida. Uma limitação nos estudos realizados até o momento reside no fato de que os limiares audiométricos dos sujeitos examinados tipicamente abrange somente a faixa central de freqüências, excluindo tanto as freqüências mais graves quanto as mais agudas audíveis por seres humanos. Por exemplo, é necessária a descrição da relação temporal entre fenômenos como o aumento do limiar de audição para altas freqüências e o aumento do limiar de resolução temporal. É necessário, portanto, determinar se o surgimento de um destes tipos de déficit é indicativo do surgimento de outros problemas auditivos próprios do envelhecimento. Este é um dos aspectos da audição que merece melhor investigação.

Para tanto, é recomendável a realização de estudos que tenham o propósito de comparar simultaneamente o desempenho de jovens e idosos com diversos graus de perda de audição em tarefas psicofísicas de resolução temporal e limiar absoluto de detecção de sons de diversas faixas de freqüência. A obtenção simultânea de ambos os conjuntos de dados permitiria verificar quais alterações ocorreriam mais precocemente, bem como verificar se há alguma correlação entre ambas.

Adicionalmente, alguns dos tópicos que estão sendo investigados na pesquisa sobre processamento temporal em si também poderiam ser examinados em sujeitos idosos como, por exemplo, os efeitos da largura das faixas de freqüência usadas como sinal e suas freqüências centrais, o efeito de filtros que permitem a audição apenas de frequiências altas ou baixas componentes de tons complexos (filtros passa-alta ou passa-baixa), o papel da integração da informação proveniente simultaneamente de vários filtros auditivos (comparação inter-canais), a comparação entre o processamento monaural e binaural de sons e a utilização de ruídos com 


\section{Processamento temporal auditivo}

freqüências diferentes antes e depois das interrupções a serem detectadas.

Adicionalmente, poderia ser verificada também, a correlação entre as alterações de processamento temporal associadas ao envelhecimento com outras características psicológicas de idosos que têm sido estudadas, como o desempenho cognitivo (Anstey \& Smith, 2000), a inteligência (Lindenberger \& Baltes, 1994), padrões de atividade cotidiana (Marsiske, Klumb, \& Baltes, 1997) e mortalidade (Anstey, Luszcz, Giles \& Andrews, 2001).

Finalmente, o estudo do processamento auditivo temporal, tem sido utilizado também, para esclarecer outros fenômenos, como a dislexia desenvolvimental (Tallal, 1986, 1993), podendo ser útil na descrição das conseqüências de danos neurológicos, como a desaferenciação auditiva decorrente da doença de Alzheimer (Sinha, Hollen, Rodriguez \& Miller, 1993).

\section{Referências}

Abel, S.M. (1972). Duration discrimination of noise and tone bursts. Journal of the Acoustical Society of America, 51, 1219-1223.

Anstey, K.J., Luszcz, M.A., Giles, L.C. \& Andrews, G.R. (2001). Demographic, health, cognitive, and sensory variables as predictors of mortality in very old adults. Psychology and Aging, 16(1), 3-11.

Anstey, K.J. \& Smith, G.A. (2000). Interrelationships among biological markers of aging, health, activity, acculturation, and cognitive performance in late adulthood. Psychology and Aging, 14(4), 605-618.

Bacon, S.P. \& Viemeister, N.F. (1985). Temporal modulation transfer functions in normal-hearing and hearing-impaired subjects. Audiology, 24, 117-134.

Chesnais, J. (1992). The demographic transition. Stages, patterns, and economic implications. Oxford: Oxford University Press.

Clark, K., Sowers, M.R., Wallace, R.B., Jannausch, M.L., Lemke, J. \& Anderson, C.V. (1995). Age-related hearing loss and bone mass in a population of rural women aged 60 to 85 years. Annals of Epidemiology, 5(1), 8-14.

Creelman, C.D. (1962). Human discrimination of auditory durations. Journal of the Acoustical Society of America, 34, 582593.

Cruickshanks, K., Wiley, T.L., Tweed, T.S., Klein, B.E.K., Klein, R., Mares-Perlman, J.A. \& Nondahl, D.M. (1998). Prevalence of hearing loss in older adults in Beaver Dam, Wisconsin: The epidemiology of hearing loss study. American Journal of Epidemiology, 148(9), 879-886.

Divenyi, P.L. \& Danner, W.F. (1977). Discrimination of time intervals marked by brief acoustic pulses of various intensities and spectra. Perception and Psychophysics, 21, 125-142.

Divenyi, P.L. \& Hirsh, I.J. (1974). Identification of temporal order in three-tone sequences. Journal of the Acoustical Society of America, 56, 144-151.

Fonda, S.J. \& Herzog, A.R. (2001). Patterns and risk factors of change in somatic and mood symptoms among older adults. Annals of Epidemiology, 11(6), 361-368.
Gates, G.A., Schmid, P., Kujawa, S.G., Nam, B.-H., D’Agostino, R. (2000). Longitudinal threshold changes in older men with audiometric notches. Hearing Research, 141, 220-228.

Giurani, F. \& Hasan, M. (2000). Abuse in elderly people: the Granny Battering revisited. Archives of Gerontology and Geriatrics, 31, 215-220.

Grose, J.H. (1991). Gap detection in multiple narrow bands of noise as a function of spectral configuration. Journal of the Acoustical Society of America, 90, 3061-3068.

Grundy, E. \& Holt, G. (2000). Adult life experiences and health in early old age in Great Britain. Social Science \& Medicine, 51, 1061-1074.

Hazzard, W.R., Woolard, N. \& Regenstreif, D. I. (2001). Internal medicine: At the nexus of the health care system in responding to the demographic imperative of an aging population. The American Journal of Medicine, 110, 507-513.

He, N.J., Horwitz, A.R., Dubno, J.R. \& Mills, J.H. (1999). Psychometric functions for gap detection in noise measured from young and aged subjects. Journal of the Acoustical Society of America, 106(2), 966-978.

Hirsh, I.J. (1959). Auditory perception of temporal order. Journal of the Acoustical Society of America, 31, 759-767.

Kacker, S.K. (1997). Hearing impairment in the aged. Indian Journal of Medical Research, 106, 333-339.

Laukkanen, P., Leskinen, E., Kauppinen, M., Sakari-Rantala, R. \& Heikkinen, E. (2000) Health and functional capacity as predictors of community dwelling among elderly people. Journal of Clinical Epidemiology, 53, 257-265.

Lee, P., Smith, J.P. \& Kington, R. (1999). The relationship of selfrated vision and hearing to functional status and well-being among seniors 70 years and older. American Journal of Ophthalmology, 127(4), 447-452.

Leinonen, R., Heikkinen, E. \& Jylhä, M. Predictors of decline in self-assessments of health among older people - a 5-year longitudinal study. Social Science \& Medicine, 52, 1329-1341.

Lindenberg, U. \& Baltes, P.B. (1994). Sensory functioning and intelligence in old age: A strong connection. Psychology and Aging, 9(3), 339-355.

Lloyd-Sherlock, P. (2000). Old age and poverty in developing countries: New policy challenges. World Development, 28(12), 21572168 .

Marsiske, M., Klumb, P. \& Baltes, M.M. (1997). Everyday activity patterns and sensory functioning in old age. Psychology and Aging, 12(3), 444-457.

McCroskey, R.L. \& Kasten, R.N. (1982). Temporal factors and the aging auditory system. Ear and Hearing, 3, 124-127.

Mills, T.L. (2001). Comorbid depressive symptomatology: isolating the effects of chronic medical conditions on self-reported depressive symptoms among community-dwelling older adults. Social Science \& Medicine, 53, 569-578.

Moore, B.C.J. (1993). Temporal analysis in normal and impaired hearing. Annals of the New York Academy of Sciences, 682, 119-136

Moore, B.C.J. (1997). An introduction to the psychology of hearing. San Diego, CA: Academic Press. 
Moore, B.C.J., Peters, R. W. \& Glasberg, B. R. (1992). Detection of temporal gaps in sinusoids by elderly subjects with and without hearing loss. Journal of the Acoustical Society of America, 92(4), 1923-1932.

Morrell, C.H., Gordon-Salant, S., Pearson, J.D., Brant, L.J. \& Fozart, J.L. (1996). Age and gender-specific reference ranges for hearing level and longitudinal changes in hearing level. Journal of the Acoustical Society of America, 100(4), Pt. 1, 1949-1957.

Pearson, J.L. \& Brown, G.K. (2000). Suicide prevention in late life: directions for science and practice. Clinical Psychology Review, Vol. 20(6), 685-705.

Penner, M.J. (1977). Detection of temporal gaps in noise as a measure of the decay of auditory sensation. Journal of the Acoustical Society of America, 61. 552-557.

Plomp, R. (1964). The rate of decay of auditory sensation. Journal of the Acoustical Society of America, 36, 277-282

Ronken, D. (1970). Monaural detection of a phase difference between clicks. Journal of the Acoustical Society of America, 47, 1091-1099.

Schneider, B.A. \& Hamstra, S.J. (1999). Gap detection thresholds as a function of tonal duration for younger and older listeners. Journal of the Acoustical Society of America, 106(1), 371-380.

Schneider, B.A., Pichora-Fuller, M.K., Kowalchuk, D. \& Lamb, M. (1994). Gap detection and the precedence effect in young and old adults. Journal of the Acoustical Society of America, 95(2), 980-991.

Schoevers, R.A., Beekman, A.T.F., Deeg, D.J.H., Geerlings, M.I., Jonker, C. \& van Tilburg, W. (2000). Risk factors for depression in later life: results of a prospective community based study (AMSTEL). Journal of Affective Disorders, 59, 127-137.

Schuknecht, H.F. (1964). Further observations on the pathology of presbycusis. Archives of Otolaryngology, 80, 369-382.

Schuknecht, H.F. (1974). Pathology of the ear. Cambridge, Ma: Harvard University Press.

Shailer, M.J. \& Moore, B.C.J. (1987). Gap detection and the auditory filter phase effects using sinusoidal stimuli. Journal of the Acoustical Society of America, 81(4), 1110-1117.

Sinha, U.K., Holen, K.M., Rodriguez, R. \& Miller, C.A. (1993). Auditory system degeneration in Alzheimer's disease. Neurology, 43 (April), 779-785.

Snell, K. B. (1997). Age related changes in temporal gap detection. Journal of the Acoustical Society of America, 101(4), 22142220.
Snell, K.B. \& Frisina, D.R. (2000). Relationships among age related differences in gap detection and word recognition. Journal of the Acoustical Society of America, 107(3), 1615-1626.

Strouse, A., Ashmead, D.H., Ohde, R.N. \& Grantham, D.W. (1998). Temporal processing in the aging auditory system. Journal of the Acoustical Society of America, 104(4), 2385-2399.

Tallal, P. (1986). Interagency Committee on Learning Disabilities: Report to the U.S. Congress: Developmental Language Disorders. Bethesda, MD: National Institute of Neurological Diseases and Stroke (NIH).

Tallal, P., Galaburda, A.M., Llinás, R. R. and Euler, C. (1993). Temporal Information Processing in the Nervous System. New York: New York Academy of Sciences.

Tsuruoka, H., Masuda, S., Ukai, K., Sakakura, Y., Harada, T. \& Majima, Y. (2001). Hearing impairment and quality of life for the elderly in nursing homes. Auris Nasus Larynx, 28, 45-54.

United Nations (1994). The sex and age distribution of the world populations. The 1994 revision. New York: UN.

Van den Berg, M.D., Oldehinkel, A.J., Brilman, E.I., Bouhuys, A. L. \& Ormel J. (2000). Correlates of symptomatic, minor and major depression in the elderly. Journal of Affective Disorders $60,87-95$.

Van den Berg, M.D., Oldehinkel, E.I., Bouhuys, A.J., Brilman, A. L., Beekman, T.F. \& Ormel J. (2001). Depression in later life: three etiologically different subgroups. Journal of Affective Disorders, 65, 19-26.

Visentin, P., Scarafiotti, C., Marinello, R., Molaschi, M. \& Fabris, F. (1998). Symptoms as predictors of functioning in the community-dwelling elderly. Archives of Gerontology and Geriatrics, 26, 247-255.

Warren, R.M. (1974). Auditory temporal discrimination by trained listeners. Cognitive Psychology, 6, 237-256.

Willott, J.F. (1991). Aging and the auditory system: Anatomy, physiology and psychophysics. San Diego, CA: Singular Publishing Group.

Willott, J.F. (1999). Neurogerontology: aging and the nervous system. New York, NY: Springer.

Wyn, R. \& Solis, B. (2001). Women's health issues across the lifespan. Women's Health Issues, 11(3), 148-159. 